\title{
Effects of factor $v$ Leiden polymorphism on the pathogenesis and outcomes of preeclampsia
}

\author{
G. K. Ababio ${ }^{1 *}$, K. Adu-Bonsaffoh² ${ }^{2}$ E. Abindau ${ }^{3}$, G. Narh ${ }^{4}$, D. Tetteh ${ }^{5}$, F. Botchway ${ }^{6}$, D. Morvey ${ }^{7}$, J. Neequaye ${ }^{8}$ and \\ I. K. Quaye ${ }^{9}$
}

\begin{abstract}
Background: Factor $V$ Leiden polymorphism is a well-recognized genetic factor in the etiology of preeclampsia. Considering that Ghana is recording high incidence of preeclampsia, we examined if factor $V$ Leiden is a contributory factor to its development and pregnancy outcomes.

Methods: STROBE consensus checklist was adopted to recruit eighty-one (81) consenting subjects after ethical clearance. Subjects were followed up till delivery to obtain outcomes of PE. Routine blood chemistry and proteinuria were done on all samples. Factor $\vee$ Leiden was characterized by polymerase chain reaction and restriction fragment length polymorphism (RFLP). The data was captured as protected health information (PHI) and analyzed with SPSS version 22.

Results: Overall allelic frequencies found in FVL exon 10 were 0.67 and 0.33 for $G$ and $A$ alleles respectively. The FVL mutation was more in PE and hypertensive patients. Increased white blood cells, increased uric acid and a three - fold increment of AST / ALT ratio was observed in PE cases when stratified by FVL exons (exon 8 and 10). Significant differences were also observed between FVL and age, systolic blood pressure (SBP), diastolic blood pressure (DBP), liver enzymes, white blood cells ( $(w b c)$, hemoglobin levels.

Conclusion: FVL mutation allele frequency was 0.33 , a first report. The mutation was associated with increased uric acid, liver enzymes and blood cell indices suggestive of acute inflammation.
\end{abstract}

Keywords: Factor V, Leiden, Preeclampsia, Polymerase chain reaction, Restriction

\section{Background}

Preeclampsia (PE) is a disease of major public health concern with high incidence of maternal and perinatal mortality [1-3]. Its etiology is multifactorial with no reliable test for predicting preeclampsia among a cohort of pregnant women. This has been complicated by the recent subclassification of preeclampsia as mild, moderate and severe or early and late onset [4]. According to the World Health Organization (WHO), maternal mortality ratio in Ghana is 350 per 100,000 live births, which is unacceptably high compared to the global ratio of 216 per 100,000 live births. Also, the maternal mortality in

\footnotetext{
* Correspondence: graceababio2002@yahoo.com

'Department of Medical Biochemistry, University of Ghana School of

Medicine and Dentistry, P. O. Box 4236, Accra, Ghana

Full list of author information is available at the end of the article
}

Korle- $\mathrm{Bu}$ Teaching Hospital (KBTH), which is the current study site, has been increasing since the mid1990s from 734.4 [5] to 915.3 per 100,000 live births in 2012 [6]. This is clearly an aggravation, so it is imperative that investigations into the etiology and pathogenesis of preeclampsia is carried out in our indigenous women to help minimize its occurrence and the associated complications. We examined if mutations in Factor V Leiden (FVL) [7], contributes to the observed upward trend in Ghana. Factor V leiden, have currently been well associated with PE development. The factor V Leiden variant resists cleavage by activated protein $C$ due to the substitution of glutamine residue with arginine at position $506\left(\mathrm{Gl}^{506}\right)$, which is the cleavage site for activated protein $C$. The resistance is seen in inherited familial thrombophilia and venous thrombosis [8] and has

(c) The Author(s). 2019 Open Access This article is distributed under the terms of the Creative Commons Attribution 4.0 International License (http://creativecommons.org/licenses/by/4.0/), which permits unrestricted use, distribution, and reproduction in any medium, provided you give appropriate credit to the original author(s) and the source, provide a link to the Creative Commons license, and indicate if changes were made. The Creative Commons Public Domain Dedication waiver (http://creativecommons.org/publicdomain/zero/1.0/) applies to the data made available in this article, unless otherwise stated. 
recently been implicated in rampant fetal loss [9]. There is therefore anticipation that evolutionary signatures such as geographical origin, mutations, cis - acting elements, trans - acting factors and natural selection might influence genetic prediction of PE and its deadly complications. The current study sought to identify factor $\mathrm{V}$ leiden mutation in PE in the Ghanaian population and examine if these are associated with $\mathrm{PE}$, to provide insight into a probable factor in the rise in $\mathrm{PE}$, seen in the general population in pregnant women attending anti-natal care at the Korle-Bu Teaching Hospital in particular.

\section{Aim}

To assess if factor $\mathrm{V}$ Leiden polymorphism contributes to preeclampsia and pregnancy outcomes in Ghanaian women.

\section{Method}

A total of ninety-six (96) subjects were recruited by simple random sampling, consisting of thirty-two (32) each of pregnant normotensive, PE and apparently healthy non pregnant women. All patients consented in writing to participate in the study, following an explanation of the aims of the study. The study was conducted at the Korle-Bu Teaching Hospital (KBTH), Accra, Ghana. Ethical approval (MS-Et/M.3 - P.3.2/2013-2014) was given by the College of Health Sciences, University of Ghana Medical School Ethical Review Board.

At recruitment, each subject completed a questionnaire (Additional file 1) after which demographic and anthropometric data were obtained. Spot urine samples were collected to determine protein-creatinine ratio using a Clinitek analytic strips. Also, 24-h urine samples were collected from PE patients, starting from the time of diagnosis, to determine the level of proteinuria. Venous blood $(5 \mathrm{ml})$ were taken from the antecubital vein into vacutainers. All pregnant women were observed till delivery, for outcomes of PE. For the purposes of this study, PE was defined based on American College of Obstetricians and Gynecologists criteria. Accordingly, PE was defined as diastolic blood pressure (DBP) of $\geq 90$ $\mathrm{mmHg}$ and or systolic blood pressure (SBP) of $\geq 140$ $\mathrm{mmHg}$ with proteinuria $\geq 300 \mathrm{mg} / \mathrm{dl}$ occurring after 20 weeks of gestation [3]. Early or late onset preeclampsia occurs before and at or after 34 weeks of gestation respectively [4]. The preeclamptic cases were managed strictly according to the standard management protocols for preeclampsia at the KBTH including the hospital admission, monitoring of maternal and fetal parameters, the use of antihypertensives and or magnesium sulphate and timely delivery of the baby.

\section{Inclusion}

For PE, only patients diagnosed with PE based on the definition were recruited after obtaining their consent. Control subjects included normotensive pregnant and non-pregnant women who gave informed consent to be included in the study.

\section{Exclusion criteria}

Patients with a history of chronic hypertension, renal disease, diabetes, urinary tract infection, cardiovascular disease, multiple pregnancy, molar pregnancy, thyroid dysfunctions and infectious diseases were excluded. Women who were less than 18 years were also excluded from the study.

\section{Sample processing}

Following the manufacturer's protocol for each reagent kit, Sysmex (XP 300 Diagnostics, Europe GmbH) automated analyzer was used to determine full blood count (FBC). The MindRay BS 240 (Shenzheni MindRay biomedical Electronics Company Ltd., Shenzheni, China) automated analyzer was used to determine lactate dehydrogenase levels (LDH), liver enzymes, BUE, creatinine, lipid profiles and uric acid levels, while URS - 10A (Wellkang Ltd., Suite B 29, Harley Street, London, UK) analytic strips were used for urine analysis.

\section{Detection of factor $\mathrm{V}$ Leiden mutations}

To determine factor $\mathrm{V}$ Leiden mutation (at exon 8 and exon 10), DNA was extracted using QIAmp mini blood kit and amplified by PCR. Following the manufacturers protocol, a total of $20 \mu \mathrm{l}$ PCR reaction containing 0.5 units of dream Taq in green Taq buffer, 2.5 ul of extracted DNAand $250 \mathrm{nM}$ of forward and reverse established primers for both exon 8 (F: CATGAGAGACATCGCCTC TG, R: GACCTAACATGTTCTAGCCAGAAG) and exon 10 (F: TGCCCAGTGCTTAACAAGACCA; R: TGTTATCACACTGGTGCTAA). The cycling conditions for amplification of exon 8 were: After initial denaturation at $95^{\circ} \mathrm{C}$ for $7 \mathrm{~min}$; 35 cycles at $94^{\circ} \mathrm{C}$ for $1 \mathrm{~min}, 51^{\circ} \mathrm{C}$ for 1 min, and $72{ }^{\circ} \mathrm{C}$ for $1 \mathrm{~min}$ and followed extension by $72{ }^{\circ} \mathrm{C}$ for $10 \mathrm{~min}$ were likewise performed. For exon 10 the cycling conditions were: initial denaturation at $94{ }^{\circ} \mathrm{C}$ for 5 min; 36 cycles at $94{ }^{\circ} \mathrm{C}$ for $1 \mathrm{~min}, 54^{\circ} \mathrm{C}$ for $1 \mathrm{~min}$, and $72{ }^{\circ} \mathrm{C}$ for $1 \mathrm{~min}$ and followed extension by $72^{\circ} \mathrm{C}$ for 10 min. For restriction analyses, $5 \mu \mathrm{l}$ of each PCR product was digested with Mnl I restriction enzyme following manufacturers' protocol and the digests were analyzed electropheretically with $3 \%$ agarose gel containing ethidium bromide.

\section{Statistical analysis}

All data were entered into an excel spread sheet (Microsoft company, USA) and analyzed using SPSS version 
18. Student's $t$ test was used to compare means of biochemical variables and analyses of variance (ANOVA) used to assess significance in the clinical variables. The dependent variable was FVL polymorphism. The independent variables included Body Mass Index (BMI), protein-creatinine ratio, proteinuria, full blood count $(\mathrm{FBC})$, lactate dehydrogenase levels (LDH), liver enzymes, BUE (Blood urea electrolytes), creatinine, lipid profiles and uric acid levels. FVL mutations within the subject groups were assessed for conformity with HardyWeinberg Equilibrium by Chi square analysis.

\section{Results}

The study was completed by eighty-one (81) subjects out of the total of 96 who were enrolled. Those who did not complete or had missing data were either ill or decided not to have their blood sample taken (Additional file 2: Table S1). The average blood pressure and weeks of maternal gestation in PE ranged from early onset $(142 / 90 \mathrm{mmHg} ; 34$ weeks $)$ to late onset $(159 / 96 \mathrm{mmHg}$, $\geq 34$ weeks) respectively. The PCR product size for exon 8 and exon 10 were $150 \mathrm{bp}$ and $276 \mathrm{bp}$ respectively. A digest in exon 8 yielded $100 \mathrm{bp}$ and $50 \mathrm{bp}$. Normal alleles in Exon 10 yielded $176 \mathrm{bp}, 69 \mathrm{bp}$ and $31 \mathrm{bp}$. Overall allelic frequencies found in FVL exon 10 were 0.67 and
Table 1 Genotypic and allelic frequency (FV exon 10)

\begin{tabular}{llll}
\hline Genotype & ctrl & BP & PE \\
\hline AA & $2(0.22)^{\mathrm{a}}$ & $5(0.20)$ & $4(0.24)$ \\
AG & $6(0.67)$ & $3(0.12)$ & $3(0.17)$ \\
GG & $1(0.11)$ & $17(0.68)$ & $10(0.59)$ \\
HW chi square test & 1.10 & 11.84 & 6.06 \\
Allelic frequency in the respective groups & & \\
G & 0.44 & 0.74 & 0.68 \\
A & 0.56 & 0.26 & 0.32
\end{tabular}

Overall Allelic frequency: $G=0.67 \quad A=0.33$

$C$ trl non-pregnant normotensive, $B P$ pregnant normotensive, $P E$ preeclampsia, $H W$ Hardy-Weinberg equilibrium

${ }^{a}$ Number (frequency)

0.33 for $\mathrm{G}$ and $\mathrm{A}$ alleles respectively (Table 1 ). There were increased FVL mutations in PE and hypertensive patients. However, the frequencies in PE and hypertensive subject were not in agreement with HardyWeinberg Equilibrium due to loss of heterozygosity. FVL homozygotes had lower age $(p<0.03)$, high systolic blood pressure, diastolic blood pressure (DBP), liver enzymes, white blood cells (wbc) and hemoglobin levels (Tables 2 and 3). The ratio of aspartate transferase (AST) to alanine transferase (ALT) had a 3-fold increase

Table 2 Exon 10 FVL stratification with clinical variables

\begin{tabular}{|c|c|c|c|c|c|c|c|c|c|c|c|c|}
\hline \multirow[t]{2}{*}{ Parameters } & \multicolumn{3}{|c|}{$\mathbf{A A}$} & \multirow[t]{2}{*}{$\mathrm{p}$-value } & \multicolumn{4}{|c|}{$\mathbf{A G}$} & \multicolumn{3}{|c|}{ GG } & \multirow{2}{*}{$\begin{array}{l}\mathrm{p}- \\
\text { value }\end{array}$} \\
\hline & CTRL & BP & PE & & CTRL & BP & PE & P-value & CTRL & $\mathrm{BP}$ & PE & \\
\hline Age (years) & $\begin{array}{l}43.00 \pm 7.07 \\
(2)\end{array}$ & $\begin{array}{l}32.00 \pm 4.76 \\
(4)\end{array}$ & $\begin{array}{l}27.75 \pm \\
4.79(4) \\
\end{array}$ & 0.0322 & $\begin{array}{l}44.00 \pm \\
14.75(6) \\
\end{array}$ & $\begin{array}{l}26.33 \pm \\
5.13(3) \\
\end{array}$ & $\begin{array}{l}36.33 \pm \\
2.31(3) \\
\end{array}$ & 0.1397 & $27(1)$ & $\begin{array}{l}30.00 \pm 4.56 \\
(18)\end{array}$ & $32.00 \pm 7.03(8)$ & 0.3919 \\
\hline $\begin{array}{l}\text { BMI } \\
\left(\mathrm{kg} / \mathrm{m}^{2}\right)\end{array}$ & $\begin{array}{l}27.42 \pm 2.81 \\
\text { (2) }\end{array}$ & $29.73(1)$ & $\begin{array}{l}24.11 \pm \\
3.06(3)\end{array}$ & 0.3822 & $\begin{array}{l}27.34 \pm 7.87 \\
(6)\end{array}$ & $\begin{array}{l}29.20 \pm \\
6.99(3)\end{array}$ & $\begin{array}{l}37.07 \pm \\
7.05(2)\end{array}$ & 0.3373 & $24.24(1)$ & $29.47 \pm$ & $30.23 \pm 7.79(7)$ & \\
\hline $\begin{array}{l}\text { SBP } \\
(\mathbf{m m H g})\end{array}$ & $108.42 \pm$ & $96(1)$ & $151.67 \pm$ & 0.0213 & $118.00 \pm$ & $97.5 \pm$ & $178(1)$ & 0.1346 & & $5.70(14)$ & & \\
\hline $\begin{array}{l}\text { (mmHg) } \\
\text { DBP } \\
(\mathrm{mmHg})\end{array}$ & $\begin{array}{l}11.20(2) \\
61.67 \pm \\
12.26(2) \\
\end{array}$ & $57.33(1)$ & \begin{tabular}{|l|}
$10.41(3)$ \\
$91.67 \pm$ \\
$10.41(3)$ \\
\end{tabular} & 0.0590 & $\begin{array}{l}15.81(6) \\
76.89 \pm \\
12.87(6) \\
\end{array}$ & $\begin{array}{l}4.01(2) \\
57.5 \pm \\
2.12(2) \\
\end{array}$ & 104(1) & 0.0904 & 114(1) & $\begin{array}{l}115.52 \pm 15.65 \\
(7)\end{array}$ & $\begin{array}{l}143.67 \pm \\
22.91(6)\end{array}$ & 0.0237 \\
\hline $\begin{array}{l}\text { Gestation at } \\
\text { diag. }\end{array}$ & & & $37(1)$ & & & & \begin{tabular}{|l|}
$31.00 \pm$ \\
$1.73(3)$ \\
\end{tabular} & & $74.5(1)$ & $\begin{array}{l}74.29 \pm 11.35 \\
(7)\end{array}$ & $\begin{array}{l}91.72 \pm \\
11.24(6)\end{array}$ & 0.0181 \\
\hline $\begin{array}{l}\text { Birth } \\
\text { weight }\end{array}$ & & & \begin{tabular}{|l}
$800.00 \pm$ \\
$435.89(3)$ \\
\end{tabular} & & & $\begin{array}{l}1901.67 \pm \\
1350.19(3) \\
\end{array}$ & & & & & $28.57 \pm 6.16(7)$ & \\
\hline $\begin{array}{l}\text { Creatinine } \\
(\mu \mathrm{mol} / \mathrm{L})\end{array}$ & $72.30(1)$ & $\begin{array}{l}54.00 \pm \\
1.13(2) \\
6.50 \pm\end{array}$ & \begin{tabular}{|l|}
52.73 \\
$\pm 16.61(3)$ \\
0.53
\end{tabular} & 0.9249 & \begin{tabular}{|l|l|}
$73.67 \pm$ \\
$21.97(4)$ \\
$1575+5.99$
\end{tabular} & $\begin{array}{l}46.13 \pm \\
4.92(2) \\
54+26(3)\end{array}$ & $\begin{array}{l}48.67 \pm \\
8.42(3) \\
4.60 \pm\end{array}$ & 0.1377 & & $\begin{array}{l}2500 \pm 1137.67 \\
(8)\end{array}$ & $\begin{array}{l}1775 \pm \\
1016.43(5)\end{array}$ & 0.2701 \\
\hline ALT (U/L) & $13.7(1)$ & $\begin{array}{l}6.50 \pm 2.83 \\
(2)\end{array}$ & \begin{tabular}{|l|}
9.53 \\
$\pm 5.22(3)$ \\
\end{tabular} & 0.5197 & \begin{tabular}{|l|}
$15.75 \pm 5.79$ \\
$(4)$ \\
\end{tabular} & $5.4 \pm 2.6(3)$ & $\begin{array}{l}4.60 \pm \\
1.98(2) \\
\end{array}$ & 0.0320 & $56.4(1)$ & $\begin{array}{l}47.81 \pm \\
16.81(8)\end{array}$ & \begin{tabular}{|l}
$73.21 \pm$ \\
$18.82(8)$
\end{tabular} & 0.0129 \\
\hline AST (U/L) & $30.7(1)$ & $12.7(2)$ & \begin{tabular}{|l|}
$30.17 \pm$ \\
$17.99(3)$ \\
\end{tabular} & & \begin{tabular}{|l|}
$20.82 \pm 4.01$ \\
$(4)$
\end{tabular} & $\begin{array}{l}11.2 \pm \\
2.62(3) \\
\end{array}$ & \begin{tabular}{|l|}
$27.00 \pm$ \\
$13.44(3)$ \\
\end{tabular} & 0.1055 & 18.4(1) & \begin{tabular}{|l}
$16.81(8)$ \\
$7.14 \pm 5.45(8)$
\end{tabular} & \begin{tabular}{|l|}
$18.82(8)$ \\
$7.11 \pm 5.16(8)$
\end{tabular} & \\
\hline $\begin{array}{l}\text { Total Chol } \\
\text { ( } \mathrm{mmol} / \mathrm{L})\end{array}$ & $5.32(1)$ & $\begin{array}{l}5.66 \pm 1.37 \\
(3)\end{array}$ & \begin{tabular}{|l}
$4.79 \pm$ \\
$2.87(3)$ \\
\end{tabular} & 0.6603 & $\begin{array}{l}5.34 \pm \\
1.77(4) \\
\end{array}$ & $\begin{array}{l}4.56 \pm \\
1.24(3)\end{array}$ & \begin{tabular}{|l}
$5.59 \pm$ \\
$2.09(3)$
\end{tabular} & 0.7565 & $30(1)$ & $12.97 \pm 3.83(8)$ & $23.62 \pm 9.41(8)$ & 0.9912 \\
\hline $\begin{array}{l}\text { TG } \\
(\mathrm{mmol} / \mathrm{L})\end{array}$ & $0.55(1)$ & $\begin{array}{l}1.40 \pm 0.19 \\
\text { (3) }\end{array}$ & $\begin{array}{l}1.87 \pm \\
0.64(3) \\
\end{array}$ & 0.2897 & $\begin{array}{l}0.92 \pm 0.70 \\
(4) \\
\end{array}$ & $\begin{array}{l}1.23 \pm \\
0.27(3)\end{array}$ & \begin{tabular}{|l|}
$1.62 \pm$ \\
$0.54(3)$ \\
\end{tabular} & 0.3222 & $3.61(1)$ & $4.97 \pm 0.97(7)$ & $5.92 \pm 1.81(8)$ & 0.0102 \\
\hline $\begin{array}{l}\text { HDL } \\
(\mathrm{mmol} / \mathrm{L})\end{array}$ & $0.62(1)$ & $\begin{array}{l}1.43 \pm 0.25 \\
\text { (3) }\end{array}$ & \begin{tabular}{|l|}
$1.30 \pm$ \\
$0.62(3)$ \\
\end{tabular} & 0.7532 & $\begin{array}{l}0.90 \pm 0.55 \\
(4)\end{array}$ & $\begin{array}{l}1.32 \pm 0.87 \\
(3)\end{array}$ & \begin{tabular}{|l|}
$1.32 \pm$ \\
$0.54(3)$ \\
\end{tabular} & 0.6304 & \begin{tabular}{|l|}
$0.63(1)$ \\
$0.71(1)$ \\
\end{tabular} & \begin{tabular}{|l|}
$2.29 \pm 0.81(7)$ \\
$1.20 \pm 0.36(7)$ \\
\end{tabular} & \begin{tabular}{|l|}
$2.07 \pm 0.69(8)$ \\
$1.53 \pm 0.35(8)$
\end{tabular} & $\begin{array}{l}0.2576 \\
0.5794 \\
0.0954\end{array}$ \\
\hline $\begin{array}{l}\text { LDL } \\
(\mathrm{mmol} / \mathrm{L})\end{array}$ & $3.19(1)$ & $\begin{array}{l}4.41 \pm 1.12 \\
\text { (3) }\end{array}$ & \begin{tabular}{|l}
$3.14 \pm 2.59$ \\
$(3)$ \\
\end{tabular} & 0.4792 & \begin{tabular}{|l}
$3.92 \pm 2.24$ \\
$(4)$ \\
\end{tabular} & $\begin{array}{l}3.51 \pm \\
0.92(3) \\
\end{array}$ & \begin{tabular}{|l|}
$3.75 \pm$ \\
$2.47(3)$ \\
\end{tabular} & 0.9659 & \begin{tabular}{|l|}
$2.38(1)$ \\
$421.3(1)$
\end{tabular} & \begin{tabular}{|l|}
$3.81 \pm 0.90(7)$ \\
$22504+5182$
\end{tabular} & $\begin{array}{l}4.49 \pm 1.69(8) \\
349.82 \pm\end{array}$ & $\begin{array}{l}0.0954 \\
0.3593 \\
\end{array}$ \\
\hline LDH (U/L) & $393.5(1)$ & \begin{tabular}{|l|}
$173.8 \pm$ \\
$17.25(3)$ \\
\end{tabular} & \begin{tabular}{|l|}
$373.90 \pm$ \\
$111.16(2)$ \\
\end{tabular} & 0.0445 & \begin{tabular}{|l|}
$317.02 \pm$ \\
$115.11(4)$ \\
\end{tabular} & $\begin{array}{l}159 \pm \\
108.31(3) \\
\end{array}$ & \begin{tabular}{|l|}
$229 \pm$ \\
$161.59(3)$ \\
\end{tabular} & 0.3253 & $421.3(1)$ & $\begin{array}{l}225.04 \pm 51.82 \\
(7)\end{array}$ & $\begin{array}{l}349.82 \pm \\
148.83(8)\end{array}$ & 0.0557 \\
\hline $\begin{array}{l}\begin{array}{l}\text { Uric Acid } \\
(\mu \mathrm{mol} / \mathrm{L})\end{array} \\
\end{array}$ & $257(1)$ & $\begin{array}{l}189.23 \pm \\
20.43(3) \\
\end{array}$ & $\begin{array}{l}329.93 \pm \\
69.20(3) \\
\end{array}$ & 0.0278 & $\begin{array}{l}208.35 \pm \\
100.29(4) \\
\end{array}$ & $\begin{array}{l}169.57 \pm \\
28.35(3) \\
\end{array}$ & $\begin{array}{l}207.34 \\
\pm 175.72(3) \\
\end{array}$ & 0.8925 & $129.4(1)$ & $\begin{array}{l}241.36 \pm \\
57.82(7)\end{array}$ & $\begin{array}{l}342.92 \pm \\
64.00(8)\end{array}$ & 0.0050 \\
\hline $\begin{array}{l}\text { Wbc } \\
\left(10^{9} / \mathbf{L}\right)\end{array}$ & $\begin{array}{l}5.86 \pm 0.60 \\
(2)\end{array}$ & $\begin{array}{l}7.09 \pm \\
1.84(5) \\
\end{array}$ & $\begin{array}{l}14.74 \pm \\
2.43(3) \\
\end{array}$ & 0.0016 & $\begin{array}{l}4.84 \pm 0.86 \\
(6) \\
\end{array}$ & $\begin{array}{l}6.62 \pm 0.40 \\
\text { (3) }\end{array}$ & $\begin{array}{l}8.34 \pm \\
3.16(2) \\
\end{array}$ & 0.0282 & & $7.99 \pm 1.37(18$ & $10.47 \pm 4.24(8)$ & 0.0320 \\
\hline $\begin{array}{l}\text { Rbc } \\
\left(10^{12} / L\right)\end{array}$ & $\begin{array}{l}4.24 \pm 0.24 \\
\text { (2) }\end{array}$ & $\begin{array}{l}3.75 \pm 0.53 \\
(5)\end{array}$ & $\begin{array}{l}4.98 \pm \\
0.44(3)\end{array}$ & 0.0076 & $\begin{array}{l}0.63 \pm 0.31 \\
(6)\end{array}$ & $\begin{array}{l}3.50 \pm \\
0.25(3)\end{array}$ & $\begin{array}{l}4.28 \pm \\
0.49(2)\end{array}$ & $<0.0001$ & & $4.08 \pm 0.58(18)$ & $4.23 \pm 0.97(8)$ & $\begin{array}{l}0.0320 \\
0.6265 \\
\end{array}$ \\
\hline $\mathrm{Hb}(\mathrm{g} / \mathrm{dL})$ & $\begin{array}{l}12.80 \pm 0.57 \\
\text { (2) }\end{array}$ & $\begin{array}{l}1.16 \\
\pm 1.39(5) \\
\end{array}$ & $\begin{array}{l}14.74 \pm \\
1.51(3) \\
\end{array}$ & 0.0236 & $\begin{array}{l}12.18 \pm 0.90 \\
(6)\end{array}$ & $\begin{array}{l}9.97 \pm 0.50 \\
\text { (3) }\end{array}$ & $\begin{array}{l}12.50 \pm \\
0.85(2)\end{array}$ & 0.0095 & & $3.57(18)$ & $12.00+2.01(0)$ & 0.3581 \\
\hline Plt $\left(10^{9} / \mathrm{L}\right)$ & $\begin{array}{l}288.5 \pm 53.03 \\
(2)\end{array}$ & $\begin{array}{l}211.40 \pm \\
79.30(5) \\
\end{array}$ & \begin{tabular}{|l}
$307.67 \pm$ \\
$103.84(3)$ \\
\end{tabular} & 0.3041 & \begin{tabular}{|l}
$286.83 \pm$ \\
$53.24(6)$ \\
\end{tabular} & $\begin{array}{l}241.67 \pm \\
29.40(3) \\
\end{array}$ & \begin{tabular}{|l|}
$175.50 \pm$ \\
$68.59(2)$ \\
\end{tabular} & 0.0631 & & $\begin{array}{l}246.00 \pm \\
77.60(18)\end{array}$ & $\begin{array}{l}232.25 \pm \\
77.21(8)\end{array}$ & 0.6799 \\
\hline $\begin{array}{l}\text { Urine } \\
\text { protein }\end{array}$ & $\begin{array}{l}5.00 \pm 0.00 \\
(2)\end{array}$ & $\begin{array}{l}11.00 \pm \\
5.48(5)\end{array}$ & $\begin{array}{l}45.00 \pm \\
49.24(3) \\
\end{array}$ & 0.2160 & $\begin{array}{l}8.33 \pm 5.15 \\
(6)\end{array}$ & $\begin{array}{l}16.67 \pm \\
12.58(3) \\
\end{array}$ & $100(1)$ & 0.1925 & & $\begin{array}{l}11.07 \pm \\
7.38(14)\end{array}$ & $\begin{array}{l}93.75 \pm \\
127.47(8)\end{array}$ & 0.0228 \\
\hline
\end{tabular}

Table 2 depicted three [3] genotypes stratified with clinical variables. Missing values in Ctrl was as expected since these women were apparently healthy and not pregnant. Missing data in other groups were those who were critically ill. Ctrl non-pregnant normotensive, BP pregnant normotensive, $P E$ preeclampsia 
Table 3 Exon 8 FVL stratification with clinical variables

\begin{tabular}{|c|c|c|c|c|c|}
\hline & Exon 8 digest & & & & \\
\hline & $\mathrm{PE}$ & Preg. normo & Non-preg. Normo & PE $\times$ Preg. normo & PE x preg. Normo x non-preg normo \\
\hline Age (years) & $30.0 \pm 6.3(7)$ & $29.3 \pm 5.0(12)$ & $44.3 \pm 7.8(10)$ & 0.7920 & $p<0.001$ \\
\hline $\mathrm{BMI}\left(\mathrm{Kg} / \mathrm{m}^{2}\right)$ & $30.2 \pm 8.5(6)$ & $28.7 \pm 3.5(9)$ & $27.7 \pm 5.6(10)$ & 0.6400 & 0.7099 \\
\hline SBP $(\mathrm{mmHg})$ & $149.5 \pm 22.6(4)$ & $99.2 \pm 4.6(4)$ & $121.4 \pm 15.8(10)$ & 0.0048 & 0.0018 \\
\hline DBP (mmHg) & $89.8 \pm 17.5(4)$ & $62.6 \pm 7.1(4)$ & $77.3 \pm 10.6(10)$ & 0.0280 & 0.0176 \\
\hline $\operatorname{ALT}(U / L)$ & $6.9 \pm 2.7(9)$ & $6.1 \pm 2.1(5)$ & $13.1 \pm 4.3(7)$ & 0.5791 & 0.0015 \\
\hline AST (U/L) & $25.4 \pm 9.8(9)$ & $12.9 \pm 2.7(5)$ & $23.9 \pm 4.7(7)$ & 0.0176 & 0.0159 \\
\hline Creatinine ( $\mu \mathrm{mol} / \mathrm{L})$ & $61.6 \pm 14.0(9)$ & $48.8 \pm 5.5(5)$ & $66.8 \pm 5.9(7)$ & 0.0770 & 0.0240 \\
\hline Total Chol (mmol/L) & $5.5 \pm 1.4(9)$ & $4.2 \pm 1.4(5)$ & $4.4 \pm 0.7(7)$ & 0.1218 & 0.1111 \\
\hline TG (mmol/L) & $1.6 \pm 0.3(9)$ & $2.3 \pm 0.8(5)$ & $0.5 \pm 0.2(7)$ & 0.0335 & $p<0.0001$ \\
\hline $\mathrm{HDL}(\mathrm{mmol} / \mathrm{L})$ & $1.5 \pm 0.4(9)$ & $0.9 \pm 0.4(5)$ & $0.7 \pm 0.3(7)$ & 0.0197 & 0.0012 \\
\hline $\mathrm{LDL}(\mathrm{mmol} / \mathrm{L})$ & $4.2 \pm 1.4(9)$ & $3.5 \pm 1.4(5)$ & $2.5 \pm 0.8(7)$ & 0.3877 & 0.0436 \\
\hline $\mathrm{LDH}(\mathrm{U} / \mathrm{L})$ & $321.7 \pm 124.6(9)$ & $222.2 \pm 50.5(5)$ & $317.0 \pm 107.8(7)$ & 0.1177 & 0.2296 \\
\hline Uric acid ( $\mu \mathrm{mol} / \mathrm{L})$ & $289.1 \pm 121.0(9)$ & $222.0 \pm 50.5(5)$ & $165.7 \pm 69.9(7)$ & 0.2655 & 0.0527 \\
\hline $\operatorname{Wbc}\left(10^{9} / \mathrm{L}\right)$ & $11.5 \pm 4.8(6)$ & $8.0 \pm 1.5(12)$ & $5.8 \pm 1.4(11)$ & 0.0309 & 0.0005 \\
\hline $\operatorname{Rbc}\left(10^{12} / \mathrm{L}\right)$ & $4.4 \pm 0.7(6)$ & $3.9 \pm 0.5(12)$ & $4.5 \pm 0.3(11)$ & 0.0985 & 0.0161 \\
\hline $\mathrm{Hb}(\mathrm{g} / \mathrm{dL})$ & $12.5 \pm 1.7(6)$ & $10.3 \pm 3.1(12)$ & $13.0 \pm 0.6(11)$ & 0.1279 & 0.0167 \\
\hline Plt $\left(10^{9} / \mathrm{L}\right)$ & $283.5 \pm 66.2(6)$ & $212.7 \pm 46.0(12)$ & $298.2 \pm 56.8(11)$ & 0.0172 & 0.0023 \\
\hline Urine Protein & $70.8 \pm 105.9(7)$ & $11.4 \pm 5.0(11)$ & $5(0)$ & 0.0769 & \\
\hline No. of preg. & $3.7 \pm 2.1(6)$ & $2.7 \pm 1.3(11)$ & & 0.2403 & \\
\hline No. of prev. Birth & $2.2 \pm 1.9(6)$ & $1.2 \pm 0.8(11)$ & & 0.1436 & \\
\hline Birth weight & $1300.0 \pm 1168.0$ & $2716.7 \pm 694.0(6)$ & & 0.0337 & \\
\hline
\end{tabular}

After multiple testing correction, age, SBP, Alt, TG, HDL, wbc and Plt remained significant. Body mass index (BMI), systolic blood pressure (SBP), diastolic blood pressure (DBP), aspartate transferase (AST), alanine transferase (ALT), total cholesterol (Total Chol), triglyceride (TG), high density lipoprotein (HDL), low density lipoprotein (LDL), lactate dehydrogenase (LDH), white blood cell (wbc), red blood cell (rbc), haemoglobin (Hb), platelet (Plt), number of pregnancies (No. of preg.), number of previous birth (No. of prev. birth)

in PE upon stratification. Also, uric acid, white blood cell count (wbc), urine protein, systolic blood pressure, diastolic blood pressure was elevated under the same stratification in PE cases while platelet count decreased (Table 2 and 3). Post hoc analysis confirmed where differences occurred between groups (Table 4, Additional file 2: Table S2).

\section{Discussion}

In this study, a report on the effects of FVL on the outcomes of PE were presented. This study was the first to report baseline FVL genotypic and allelic frequencies as well as relating clinical variables with it. The study also reports for the first time that in the pregnant women studied, FVL mutation allele frequency being 0.33 , is consistent with reports elsewhere [9]. The loss of heterozygosity in the PE and hypertensive subjects could be due to founder effects or genetic drifts [10]. These could be critically examined in a follow up study.

The strikingly three - fold increment of AST / ALT ratio, increased white blood cell (wbc) and increased uric acid levels observed in PE cases when stratified by both exons indicates that genetic disposition to FVL could be associated with susceptibility to PE and might confer poor PE outcomes. The underlying pathophysiology appears to be acute inflammation as all blood cell indices were elevated including uric acid. These were also consistent with previous findings in other populations [11-14].

Although, wbc counts [11-14], liver enzymes [11-14] and uric acid [15-19] had been suggested to increase in $\mathrm{PE}$, it was notable to mention that no study associated FVL to such parameters in Ghana. More so, there had been scanty information on the impact of FVL in clinical events where inflammation was present in the country. FVL referred to a specific gene mutation which led to hypercoagulability with serious clinical consequences.

Much work has been done elsewhere on FVL [20-26] in PE; however, while others never found FVL mutation in their cohort [23], others strongly cited indications of FVL [22, 24] being a unique predictor of PE. Even though sample size in the current study was small, findings of heterozygosity and normal homozygous in FVL 
Table 4 Multiple Comparisons FV exon 10

\begin{tabular}{|c|c|c|c|c|c|c|c|}
\hline & \multirow{2}{*}{$\begin{array}{l}\text { (I) } \\
\text { idCat }\end{array}$} & \multirow{2}{*}{$\begin{array}{l}(\mathrm{J}) \\
\text { idCat }\end{array}$} & \multirow{2}{*}{$\begin{array}{l}\text { Mean } \\
\text { Difference } \\
(I-J)\end{array}$} & \multirow{2}{*}{$\begin{array}{l}\text { Std. } \\
\text { Error }\end{array}$} & \multirow[t]{2}{*}{ Sig. } & \multicolumn{2}{|c|}{ 95\% Confidence Interval } \\
\hline & & & & & & Lower Bound & Upper Bound \\
\hline \multirow[t]{6}{*}{ Tukey HSD } & \multirow[t]{2}{*}{1.00} & 2.00 & $-.921^{*}$ & .277 & .010 & -1.62 & -.22 \\
\hline & & 3.00 & $-.810^{*}$ & .306 & .040 & -1.59 & -.03 \\
\hline & \multirow[t]{2}{*}{2.00} & 1.00 & $.921^{*}$ & .277 & .010 & .22 & 1.62 \\
\hline & & 3.00 & .111 & .290 & .923 & -.63 & .85 \\
\hline & \multirow[t]{2}{*}{3.00} & 1.00 & $.810^{*}$ & .306 & .040 & .03 & 1.59 \\
\hline & & 2.00 & -.111 & .290 & .923 & -.85 & .63 \\
\hline \multirow[t]{6}{*}{ Bonferroni } & \multirow[t]{2}{*}{1.00} & 2.00 & $-.921^{*}$ & .277 & .011 & -1.65 & -.19 \\
\hline & & 3.00 & $-.810^{*}$ & .306 & .048 & -1.61 & -.01 \\
\hline & \multirow[t]{2}{*}{2.00} & 1.00 & $.921^{*}$ & .277 & .011 & .19 & 1.65 \\
\hline & & 3.00 & .111 & .290 & 1.000 & -.65 & .87 \\
\hline & \multirow[t]{2}{*}{3.00} & 1.00 & $.810^{*}$ & .306 & .048 & .01 & 1.61 \\
\hline & & 2.00 & -.111 & .290 & 1.000 & -.87 & .65 \\
\hline \multirow[t]{6}{*}{ Tamhane } & \multirow[t]{2}{*}{1.00} & 2.00 & $-.921^{*}$ & .205 & .002 & -1.48 & -.36 \\
\hline & & 3.00 & -.810 & .363 & .174 & -1.95 & .33 \\
\hline & \multirow[t]{2}{*}{2.00} & 1.00 & $.921^{*}$ & .205 & .002 & .36 & 1.48 \\
\hline & & 3.00 & .111 & .364 & .988 & -1.03 & 1.25 \\
\hline & \multirow[t]{2}{*}{3.00} & 1.00 & .810 & .363 & .174 & -.33 & 1.95 \\
\hline & & 2.00 & -.111 & .364 & .988 & -1.25 & 1.03 \\
\hline
\end{tabular}

ANOVA at $95 \% \mathrm{Cl}$, significance $=0.009, \mathrm{~F}=6.131$

Dependent variable: FV exon 10

Factor: ID catr (ID categories)

Number of samples $=57$

Strata variables: age categories, BMI categories, SBP categories, DBP categories, Uric acid categories, wbc categories, LDH categories

1 = non-pregnant normotensive 2 = pregnant normotensive 3 = preeclampsia

exon 10 seemed to be consistent with Dizon - Townson et al. [9] We could not however extrapolate the findings to the general population unless this was further interrogated with a larger sample size. We tried to minimize selection bias using two control(s) e.g. pregnant normotensive and non-pregnant normotensive per case to improve the statistical power of the study since those from the hospital setting had outcomes related to the exposure being studied.

The inflammatory response attributed to FVL might be due to platelets activating the inflammation, with uric acid channeling macrophage and dendritic cell activation, thereby increasing proinflammatory cytokines to sustain inflammation and adverse outcomes [25]. The absence of FVL mutation in exon 8 was consistent with O'Shaughnessy et al. [26] Nevertheless, the current study was able to identify mutant homozygous in FVL exon 10.

\section{Conclusion}

FVL mutation could lead to an inflammatory state in PE. We have for the first time reported the allelic frequency in a section of the Ghanaian population.

\section{Supplementary information}

Supplementary information accompanies this paper at https://doi.org/10. 1186/s12881-019-0924-6.

Additional file 1:. Questionnaire

Additional file 2: Table S1. Performance profile of the Preparative Technique(s) used. Table S2. Bootstrap for Multiple Comparisons: Dependent Variable: FV exon 10

\section{Abbreviation(s)}

ALT: Alanine transferase; AST: Aspartate transferase; FVL: Factor V Leiden; Gln: Glutamine; Hb: Haemoglobin; HDL: High density lipoprotein; KBTH: Korle-Bu Teaching Hospital; LDH: Lactate Dehydrogenase; LDL: Low density lipoprotein; PCR: Polymerase Chain Reaction; PE: Preeclampsia; Plt: Platelet; rbc: red blood cell; Total Chol: Total Cholesterol; Tri: Triglyceride; wbc: white blood cell; WHO: World Health Organization

\section{Acknowledgements}

The authors acknowledge Mensah J, Amponsah C, staff of Obstetrics and Gynecology of the Korle-Bu Teaching Hospital, Accra, Ghana; and also, Medical Biochemistry, Univ. of Ghana SBAHS and ORID, University of Ghana, Legon.

Availability of data materials

The datasets generated and analyzed could be found in the Mendeley repository (DOI: https://doi.org/10.17632/zvbbtby75p.4). https://data. mendeley.com/datasets/zvbbtby $75 \mathrm{p} / 4$ 


\section{Funding disclosure}

Work from the authors' laboratory was supported by grants from ORID, University of Ghana, Legon and additional soft grant from QI. The funders had no role in data collection, study design, data analysis, preparation of manuscript and or decision to publish.

\section{Authors' contributions}

Conceptualization, Methodology, Supervision and Writing (review and editing) were by AGK, ABK, QI. Original draft and funding acquisition were by AGK. QI and AGK provided resources. Experimental Investigation: AGK, MD, TD, BF, NG, AE, NJ. Formal Data Analysis: AGK, AE, NG. All authors have read and approved the manuscript.

\section{Ethics approval and consent to participate}

Before the study was implemented, permission was sought from the University of Ghana Medical School Ethical and protocol unit (MS-Et/M.3 P.3.2/2013-2014). The protocol number had been included in the methods section of this manuscript. Consent for participation in the study was obtained in the language chosen by the participant. Any potential illiterate subject had the consent form translated in the language of his /her choice and consent recorded by a witnessed ' $X$ " on the consent form. Emphasis was placed on the fact that a subject was free to participate or decline without any effect on his/her medical care.

\section{Consent for publication}

Not Applicable.

\section{Competing interests}

The authors declare that they have no competing interests.

\section{Author details}

${ }^{1}$ Department of Medical Biochemistry, University of Ghana School of Medicine and Dentistry, P. O. Box 4236, Accra, Ghana. ${ }^{2}$ Department of Obstetrics and Gynecology, University of Ghana School of Medicine and Dentistry, Accra, Ghana. ${ }^{3}$ Department of Physiology, University of Ghana School of Medicine and Dentistry, Accra, Ghana. ${ }^{4}$ Department of Biochemistry, University of Ghana, Accra, Ghana. ${ }^{5}$ National Diabetic Research Lab, Korle-Bu Teaching Hospital, Accra, Ghana. ${ }^{6}$ Department of Child Health, Korle-Bu Teaching Hospital, Accra, Ghana. ${ }^{7}$ Clinical Documentation Improvement Specialist, Southern California Hospital, Culver City, CA 90232, USA. ${ }^{8}$ Biochemistry, Kwame Nkrumah University of Science And Technology, Kumasi, Ghana. ${ }^{9}$ Department of Applied Health Sciences, Regent University of Science and Technology, Accra, Ghana.

\section{Received: 14 December 2018 Accepted: 12 November 2019}

\section{Published online: 27 November 2019}

\section{References}

1. Irminger-Finger I, Jastrow N, Irion O. Preeclampsia: A danger growing in disguise. Int J Biochem Cell Biol. 2008;40:1979-83.

2. Saadat M, Nejad SM, Habibi G, Sheikhvatan M. Maternal and neonatal outcomes in women with preeclampsia. Taiwan J Obstet Gynecol. 2007;46: 255-9.

3. American College of Obstetrician and Gynaecologis. Diagnosis and management of preenclampsia and eclampsia. ACOG Practice Bulletin 2002; No. 33. Washington DC.

4. Raymond D, Peterson E. A critical review of early onset and late-onset preeclampsia. Obstet Gynecol Surv. 2011;66(8):497-506.

5. Lassey AT, Wilson JB. Trends in maternal mortality in Korle Bu Teaching Hospital, 1984-1994. Ghana Med J. 1998;32a:910-6.

6. Adu-Bonsaffoh K, Oppong SA, Binlinla G, \& Obed SA. Maternal deaths attributable to hypertensive disorders in a tertiary hospital in Ghana. International Journal of Gynecology \& Obstetrics 2013.

7. Tranquilli AL, Emanuelli M. The thrombophilic fetus. Med Hypotheses. 2006; 67(5):1226-9.

8. Frosst P, Blom HJ, Milos R, Goyette P, Sheppard CA, Matthews RG, Boers $\mathrm{GJH}$, van den Heuvel LP, Rozen R. A candidate genetic risk factor for vascular disease: a common mutation in methylenetetrahydrofolate reductase. Nat Genet. 1995;10:111-3.
9. Dizon-Townson DS, Nelson LM, Easton K, Ward K. The factor V Leiden mutation may predispose women to severe preeclampsia. Am J Obstet Gynecol. 1996;175(4):902-5.

10. Rees DC. The population genetics of factor V Leiden (review). Brit J Haem. 1996;95:579-86.

11. Rinehart BK, Terrone DA, Lagoo-Deenadayalan S, Barber WH, Martin JN, Bennett WA. Expression of the placental cytokines tumor necrosis factor a interleukin $1 \beta$, and interleukin 10 is increased in preeclampsia. Am J Obstet Gynecol. 1999;181(4):915-20.

12. Sibai BM. Diagnosis, controversies, and management of the syndrome of hemolysis, elevated liver enzymes, and low platelet count. Obstet Gynecol. 2004;103(5, Part 1):981-91.

13. Sibai BM, Taslimi MM, El-Nazer A, Amon E, Mabie BC, Ryan GM. Maternal-perinatal outcome associated with the syndrome of hemolysis, elevated liver enzymes, and low platelets in severe preeclampsiaeclampsia. Am J Obstet Gynecol. 1986;155(3):501-7.

14. Sibai BM. The HELLP syndrome (hemolysis, elevated liver enzymes, and low platelets): much ado about nothing? Am J Obstet Gynecol. 1990;162(2):311-6.

15. Bainbridge SA, Roberts JM. Uric acid as a pathogenic factor in preeclampsia. Placenta. 2008;29:67-72.

16. Powers RW, Bodnar LM, Ness RB, Cooper KM, Gallaher MJ, Frank MP, Daftary $A R$, Roberts JM. Uric acid concentrations in early pregnancy among preeclamptic women with gestational hyperuricemia at delivery. Am J Obstet Gynecol. 2006;194(1):160-e1.

17. Roberts JM, Bodnar LM, Lain KY, Hubel CA, Markovic N, Ness RB, Powers RW. Uric acid is as important as proteinuria in identifying fetal risk in women with gestational hypertension. Hypertension. 2005;46(6):1263-9.

18. Johnson RJ, Kang DH, Feig D, Kivlighn S, Kanellis J, Watanabe S, Tuttle KR, Rodriguez-Iturbe B, Herrera-Acosta J, Mazzali M. Is there a pathogenetic role for uric acid in hypertension and cardiovascular and renal disease? Hypertension. 2003;41(6):1183-90.

19. Ababio GK, Adu-Bonsaffoh K, Botchway F, Abindau E, Quaye IKE. Hyperuricemia and adverse pregnant outcomes in Ghanaian women: potential mechanism. Biochem Anal Biochem. 2016;5:2.

20. Grandone E, Margaglione M, Colaizzo D, Cappucci G, Paladini D, Martinelli P, Montanaro S, Pavone G, Di GM. Factor V Leiden, C> T MTHFR polymorphism and genetic susceptibility to preeclampsia. Thromb Haemost. 1997;77(6):1052-4

21. Younis JS, Ben-Ami MO, Brenner B, Ohel G, TAL J. Activated protein C resistance and factor $\vee$ Leiden mutation can be associated with first-as well as second-trimester recurrent pregnancy loss. Am J Reprod Immunol. 2000; 43(1):31-5.

22. Rigo J Jr, Rigó J, Nagy B, Fintor L, Tanyi J, Beke A, Karádi I, Papp Z. Maternal and neonatal outcome of preeclamptic pregnancies: the potential roles of factor $\vee$ Leiden mutation and 5, 10 methylenetetrahydrofolate reductase. Hypertension in pregnancy. 2000;19(2):163-72.

23. Rosendaal FR, Koster T, Vandenbroucke JP, Reitsma PH. High risk of thrombosis in patients homozygous for factor $\mathrm{V}$ Leiden (activated protein $\mathrm{C}$ resistance). Blood. 1995;85(6):1504-8.

24. Nafa K, Bessler M, Mason P, Vulliamy T, Hillmen P, Castro-Malaspina H, Luzzatto L. Factor $V$ Leiden mutation investigated by amplification created restriction enzyme site (ACRES) in PNH patients with and without thrombosis. Haematologica. 1996;81(6):540-2.

25. Perez-Pujol S, Aras O, Escolar G. Factor V Leiden and inflammation. Thrombosis. 2012;2012.

26. O'Shaughnessy KM, Fu B, Ferraro F, Lewis I, Downing S, Morris NH. Factor V Leiden and Thermolabile Methylenetetrahydrofolate Reductase gene variants in an east Anglian preeclampsia cohort. Hypertension. 1999;33:1338-41.

\section{Publisher's Note}

Springer Nature remains neutral with regard to jurisdictional claims in published maps and institutional affiliations. 